\title{
Geregelter Meßplatz für Sensoren zur invasiven Blutdruckmessung
}

\author{
El-Natsheh N., Zerle S., Köhler B., Orglmeister R.
}

Technische Universităt Berlin, Institut für Elektronik, Einsteinufer 17/EN 3, D-10587 Berlin

\section{EINLEITUNG}

Invasive Blutdruckmessungen ermöglichen eine kontinuierliche Registrierung des Blutdruckverlaufes und sind heute unentbehrlich bei Diagnostik und Therapie in Anästhesie und Intensivmedizin. Die Anzahl der jährlich durchgeführten invasiven Blutdruckmessungen wird in Europa auf etwa zweieinhalb, in den USA auf acht Millionen geschätzt [1].

Eine genaue und zuverlässige Wiedergabe des Blutdrucksignals durch das Meßsystem ist eine Voraussetzung für die Effizienz einer nachfolgenden ärztlichen Behandlung. Fehlereinflüsse der Meßkette können durch Optimierung und Abstimmung ihrer Komponenten schon während der Entwicklungsphase minimiert werden. Dabei spielt neben der theoretischen Berechnung die praktische Erprobung eine große Rolle. Da klinische Untersuchungen nicht immer möglich sind, werden leistungsfähige Blutdrucksimulatoren benötigt.

Mit dem gegenwärtig auf dem Markt erhältlichen Blutdrucksimulator [2] kann die Einhaltung der von der Association for Advancement of Medical Instrumentation (AAMI) [3] festgelegten Anforderungen an Druckmessungen nicht in allen Punkten ausreichend geprïft werden. Dies betrifft insbesondere den Druckmeßbereich und die Temperierung des Mediums [2].

Der im vorliegenden Artikel vorgestellte neuartige $\mathrm{Si}$ mulator ist ein kompletter Meßplatz für die Prüfung der gesamten Blutdruckmeßkette entsprechend den AAMISpezifikationen für alle gängigen medizinischen Drucksensoren [4,5]. Mit Hilfe einer mikrocontrollergesteuerten Einheit werden gespeicherte technische Solldruckkurven einstellbarer Amplitude und Frequenz sowie physiologische Druckverläufe erzeugt und die Erfassung der Meßdaten vorgenommen. Über eine serielle Schnittstelle können darüber hinaus beliebige Solldruckkurven von und Meßdaten zu einem Personalcomputer übertragen und dort ausgewertet werden. Für möglichst realitätsnahe Messungen wurden zusätzlich ein Spülsystem sowie eine Flußsimulation implementiert.

\section{REALISIERUNG}

Den prinzipiellen Aufbau des geregelten Meßplatzes zeigt Abbildung 1. Er setzt sich aus drei Kernbereichen zusammen: (1) einem Mikrocontroller mit zentralen Steuerungs- und Überwachungsaufgaben, (2) den elektromechanischen und mechanischen Komponenten sowie (3) dem über eine serielle Schnittstelle verbundenen Personalcomputer. Die Realisierung der zentralen Steue- rung des Blutdrucksimulators erfolgt mit Hilfe des 32 Bit-Mikrocontrollers MC68332. Er ist zuständig für die Benutzerschnittstelle am Gerät sowie die Kommunikation mit dem externen Personalcomputer. Darüber hinaus kontrolliert der Mikrocontroller alle wesentlichen Funktionen des Gerätes, d.h. Fluß, Temperatur, Spülsystem und die Versorgung der Drucksensoren sowie die Druckerzeugung und die Aufnahme der Meßwerte. Die Auflösung der generierten Drucksignale und der aufgenommenen Meßwerte beträgt 12 Bit bei einer Abtastfrequenz von $1000 \mathrm{~Hz}$. Durch verschiedene Störfaktoren bedingt entspricht dies einer Auflösung von $1 \mathrm{mmHg}$.

Das Prüfmedium (physiologische $\mathrm{NaCl}-\mathrm{Lösung}$ ) des Simulators befindet sich in einem geschlossenen Kreislauf. Als Sensorzugang stehen zwei Lure-Lock-Anschlüsse zur Verfügung. Für hydrostatische Druckmessungen ist

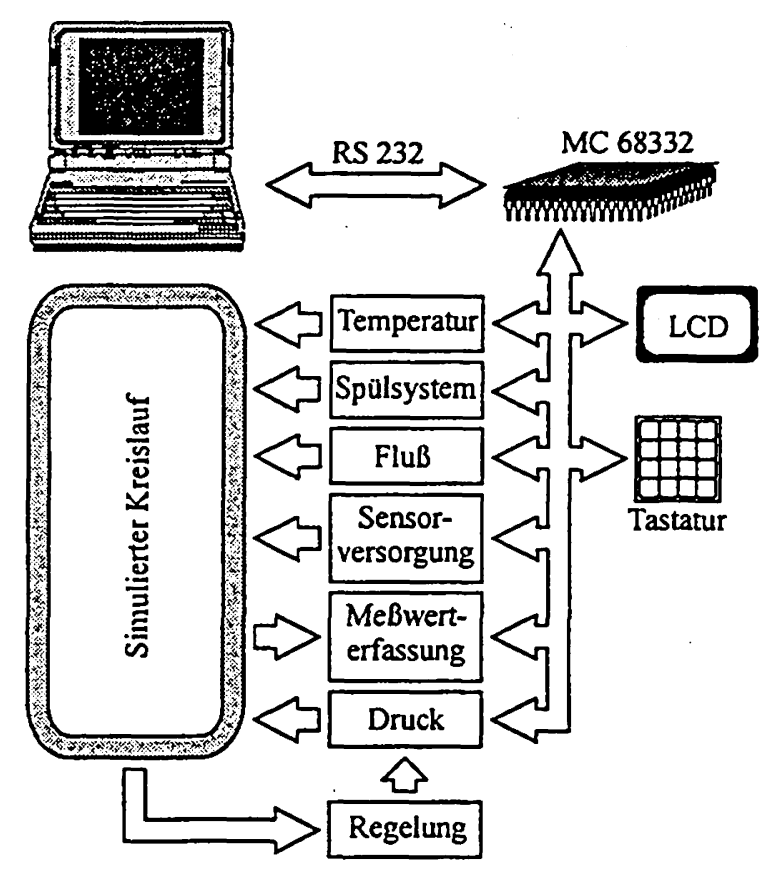

\section{Abbildung 1: Prinzip des geregelten Meßplatzes}

ein Zugang senkrecht zur FluBrichtung angebracht, der zweite ermöglicht einen Katheterzugang unter einem Winkel von $35^{\circ}$ für Gesamtdruckmessungen. Der Aufbau des Kreislaufs aus Plexiglas bietet die Möglichkeit, Luftblasen im Kreislauf zu erkennen. Gegenüber Metallen ist außerdem die geringe Wärmeleitfähigkeit von $0,184 \mathrm{~W} /(\mathrm{mK})$ und die $\mathrm{NaCl}$-Beständigkeit vorteilhaft.

Das Prüfmedium kann auf physiologische Temperaturen erwärmt werden. Zur Temperierung stehen zwei Heiz- 
elemente zur Verfügung. Temperaturwerte zwischen Raumtemperatur und $45^{\circ} \mathrm{C}$ sind in $1^{\circ} \mathrm{C}$-Schritten einstellbar. Um die Erwärmungszeit beim Start einer Messung auf die gewünschte Temperatur kurz zu halten, werden in der Initialisierungsphase zwei Heizungen unterschiedlicher Leistung eingeschaltet. Bei Erreichen der Solltemperatur wird die leistungsstärkere automatisch ausgeschaltet und nur noch mit der leistungsschwächeren Heizung nachgeregelt. Mit Hilfe einer Kreiselpumpe wird die $\mathrm{NaCl}$-Lösung pulsationsarm mit einstellbarer Flußrate umgewälzt und somit eine nahezu gleichmäßige Verteilung der Wärme erreicht.

Der Prüfling kann praxisnah an den Kreislauf und an ein integriertes Spülsystem angeschlossen werden. $\mathrm{Da}$ der simulierte Blutkeislauf ein geschlossenes System darstellt, würde der Anschluß eines handelsüblichen Spülsystems eine stetige Steigerung des Mitteldrucks bewirken. Es wurde aus diesem Grunde ein Spülsystem entwickelt, das dem Kreislauf Flüssigkeit zur Spülung entnimmt und die entnommene Flüssigkeitsmenge anschließend wieder in den Kreislauf zurückführt.

Für die Druckerzeugung findet eine Tauchspule Verwendung. Über einen Metallbalg wird die stromproportionale Kraft der Tauchspule auf das im Kreislauf eingeschlossene Prüfmedium übertragen. Der Metallbalg besitzt eine große Flexibilität in Richtung der Kraftwirkungsline und zeichnet sich im Vergleich zu anderen Lösungen [2] durch eine hohe querwirkende Steifigkeit und geringe Massenträgheit aus. Damit können sowohl positive als auch negative Drücke verlustarm generiert werden. Da das Prüfmedium nahezu inkompressibel ist, bewirkt schon eine geringfügige Auslenkung eine erhebliche Druckänderung. Daher arbeitet die Kombination von Tauchspule und Metallbalg immer im linearen Bereich. Die Erzeugung der Druckkurven im Bereich von -100 bis $+300 \mathrm{mmHg}$ ist auf diese Weise problemlos möglich. Eine zusätzliche Erhöhung der Genauigkeit des erzeugten Druckverlaufs wird durch die implementierte Regelung mit Hilfe eines analogen PI-Reglers erreicht.

Die Speisung der Tauchspule erfolgt durch eine spannungsgesteuerte Präzisionsstromquelle. Somit haben Änderungen des ohmschen Widerstandes der Spule aufgrund von Temperaturschwankungen keinen Einfluß auf den erzeugten Druck. Der Permanentmagnet ist so dimensioniert, daß Ströme unterhalb von einem Ampère zur Generierung des Drucks im gesamten Arbeitsbereich genügen. Aufgrund der geringen Massenträgheit der Spule können sehr hohe Flankensteilheiten erzielt werden.

Die für verschiedene Sensoren unterschiedlichen Versorgungsspannungen können menügeführt am Gerät eingestellt werden, so daß keine zusätzlichen externen Spannungsquellen erforderlich sind.

Zur Aufbereitung, Darstellung und Speicherung der Soll- und Meßdruckkurven wurde eine PC-Oberfläche entwickelt, die einen bidirektionalen Datenaustausch $\mathrm{zwischen}$ dem Blutdrucksimulator und einem PC über die RS232-Schnittstelle ermoglicht.
Abbildung 2 zeigt den fertigen Aufbau des Blutdrucksimulators.

\section{ZUSAMMENFASSUNG}

Es wurde ein vollständiger Meßplatz für die Validierung von Anordnungen der invasiven Blutdruckmessung entsprechend den Spezifikationen der AAMI entwickelt.

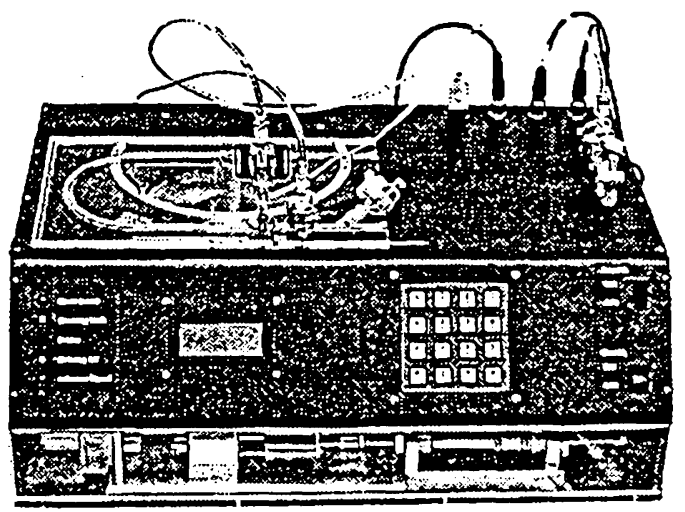

\section{Abbildung 2: Geregelter Blutdrucksimulator}

Das mikrocontrollergesteuerte Gerät ist in der Lage, beliebige technische, physiologische und pathologische Solldruckkurven zu erzeugen und Druckmeßdaten zu liefern. Eine nachträgliche Datenauswertung wird durch eine Schnittstelle zu einem Personalcomputer ermöglicht.

\section{DANKSAGUNG}

Wir möchten uns an dieser Stelle für die vielen anregenden Diskussionen mit den Mitarbeitern des Institutes für Elektronik der Technischen Universität Berlin, der B. Braun Melsungen AG und der Bundesanstalt für Materialforschung und -prüfung bedanken.

\section{LITERATUR}

[1] Gilly H., Fizal S.: Prinzip und Praxis in der invasiven arteriellen Druckmessung. Anaesthesist (1995) 44: 931-952, Springer Verlag

[2] BIO-TEK Blood Pressure System Calibrator, Model 601A Operators's Manual, Manual Part Number 94016, Revision E, 1987

[3] American National Standard for Blood Pressure Transducers, General. Arlington, USA, AAMI, 1986

[4] El-Natsheh N.: Entwurf, Aufbau und Test elektromechanischer Komponenten eines geregelten Blutdrucksimulators. Diplomarbeit. Technische Universität Berlin, Institut für Elektronik, März 1998

[5] Zerle S.: Entwurf, Aufbau und Test elektronischer Komponenten eines geregelten Blutdrucksimulators. Diplomarbeit, Technische Universität Berlin, Institut für Elektronik, März 1998 